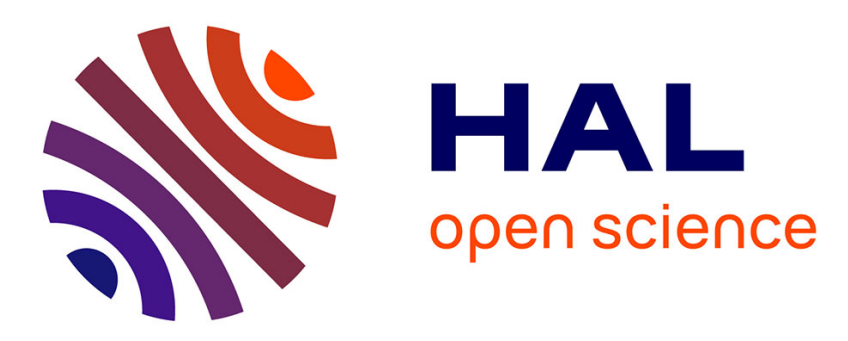

\title{
Analytical methods to support continuous improvements at Scania
}

Marcello Colledani, Michael Ekvall, Thomas Lundholm, Paolo Moriggi, Andrea Polato, Tullio Tolio

\section{- To cite this version:}

Marcello Colledani, Michael Ekvall, Thomas Lundholm, Paolo Moriggi, Andrea Polato, et al.. Analytical methods to support continuous improvements at Scania. International Journal of Production Research, 2010, 48 (07), pp.1913-1945. 10.1080/00207540802538039 . hal-00565118

\section{HAL Id: hal-00565118 https://hal.science/hal-00565118}

Submitted on 11 Feb 2011

HAL is a multi-disciplinary open access archive for the deposit and dissemination of scientific research documents, whether they are published or not. The documents may come from teaching and research institutions in France or abroad, or from public or private research centers.
L'archive ouverte pluridisciplinaire HAL, est destinée au dépôt et à la diffusion de documents scientifiques de niveau recherche, publiés ou non, émanant des établissements d'enseignement et de recherche français ou étrangers, des laboratoires publics ou privés. 


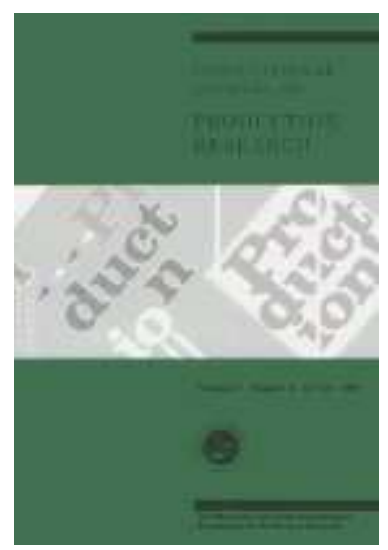

Analytical methods to support continuous improvements at Scania

\begin{tabular}{|r|l|}
\hline Journal: & International Journal of Production Research \\
\hline Manuscript ID: & TPRS-2008-IJPR-0365.R1 \\
\hline Manuscript Type: & Original Manuscript \\
\hline Date Submitted by the \\
Author: & $26-$ Sep-2008 \\
\hline Complete List of Authors: & $\begin{array}{l}\text { Colledani, Marcello; Politecnico di Milano, Mechanical Engineering } \\
\text { Ekvall, Michael; Scania CV AB } \\
\text { Lundholm, Thomas; KTH - Kungliga Tekniska hogskolan, Design } \\
\text { and Management of Manufacturing Systems } \\
\text { Moriggi, Paolo; Politecnico di Milano, Mechanical Engineering } \\
\text { Polato, Andrea; Politecnico di Milano, Mechanical Engineering } \\
\text { Tolio, Tullio; Politecnico di Milano, Mechanical Engineering }\end{array}$ \\
\hline Keywords: & $\begin{array}{l}\text { PRODUCTIVITY IMPROVEMENT, PERFORMANCE ANALYSIS, } \\
\text { PRODUCTION FLOW ANALYSIS, TRANSFER LINES, BUFFER SIZING }\end{array}$ \\
\hline Keywords (user): & \begin{tabular}{l} 
PRODUCTIVITY IMPROVEMENT, PERFORMANCE ANALYSIS \\
\hline
\end{tabular} \\
\hline
\end{tabular}

\section{scholarONE" \\ Manuscript Central}




\title{
RESEARCH ARTICLE
}

\section{Analytical methods to support continuous improvements at Scania}

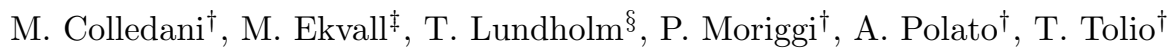 \\ (Received 00 Month 200x; final version received 00 Month 200x)
}

\begin{abstract}
Considerable effort is usually devoted by companies to ensure the competitiveness of their manufacturing systems. This makes continuous improvement a central topic in production management activities. Less attention is given to the methods that drive improvement and to the way actions are defined and selected. In the literature, analytical models and simulation are the most widely known tools for manufacturing systems performance evaluation and improvement. In practice, simple approaches, mainly based on experience, seems to be the best compromise to face this problem. In a collaboration project among Politecnico di Milano (Milano, Italy), Kungliga Tekniska högskolan (Stockholm, Sweden) and Scania CV AB (Södertälje, Sweden) within the European Network of Excellence VRL-KCiP we proposed a new methodology, based on analytical methods, to support the company in the manufacturing system productivity improvement through re-configuration. The application of this approach to the Scania 6-cylinder engine-block machining line enabled a remarkable increment in throughput by selecting analytically the most suitable improvement actions.
\end{abstract}

\section{Introduction}

Different tools and methodologies have been proposed in the literature to cope with manufacturing system performance evaluation, above all being simulation and analytical tools (Nyhuis and Wiendahl 2006). Simulation has the advantage of being flexible and is able to model a wide set of systems, at the desired level of detail. It is generally suggested as an off-line tool to be used to evaluate the performance of manufacturing systems and identify existing improvement possibilities. Due to the difficulty in building and validating the simulation model, the complexity in defining the proper degree of detail of the model and the long computing times needed to obtain significant results, simulation is mainly used during the last steps of the manufacturing system configuration/reconfiguration process. Indeed, in this phase only a few feasible alternatives among which to select the best solution are considered (DeVin et al. 2004). Examples of simulation approaches adopted for estimating the main performance measures in production systems and for supporting their design can be found in Savsar and Al-Jawini (1995), Phillis et al. (1997), Bley and Wuttke (1997).

Analytical tools have been developed with the aim of evaluating the production system performance by proposing mathematical modeling of the system dynamic behaviour. They are useful in understanding the relations among different system variables, which are explicitly described by means of equations. Thanks to their quickness, analytical methods are particularly suitable during the early configura-

\footnotetext{
${ }^{\dagger}$ Politecnico di Milano, Department of Mechanical Engineering - Manufacturing and Production Systems Division. Via La Masa 1, 20156 Milano, Italy.

¥Scania CV AB, SE-151 87 Södertälje, Sweden.

$\S$ Kungliga Tekniska högskolan, Design and Management of Manufacturing Systems. Brinellvägen 68, SE-
} 10044 Stockholm, Sweden.

ISSN: 0020-7543 print/ISSN 1366-588X online (C) 200x Taylor \& Francis DOI: $10.1080 / 00207540 \mathrm{xxxxxxxxx}$ http://www.informaworld.com 
tion phase of a manufacturing system, when a large number of alternatives has to be evaluated in a short time. Their analytical nature also allows identifying the system modifications that have higher impact on the system performance. Moreover, by coupling the performance evaluation methods with proper optimization algorithms it is eventually possible to determine the right setting of the system parameters, resulting in adherence to the performance requirements according to the configuration constraints. The main drawback of these methods is the fact that, in order to guarantee mathematical tractability of the problem, they are based on restrictive assumptions that are hardly ever observed in real systems. One example is the commonly adopted infinite buffer capacity assumption. While dealing with production systems characterized by long and complex layouts and flow management rules, existing analytical methods fail to be applicable since the modeling assumptions are no more verified.

To overcome this limitation, in the last two decades, much effort has been dedicated by researchers in the development and validation of approximate analytical methods, based on the decomposition technique, for evaluating the performance of complex production systems. Existing techniques allow to model and analyse a very wide range of real systems and production management policies, with high accuracy in the approximation, when compared to exact methods (estimation errors are commonly lower than $5 \%$ ). Given the uncertainty in real factory data these models take as input, the estimation error provided by the application of these methods does not compromise their practical use. The results presented in Dallery et al. (1988) and Gershwin (1994) can be considered as the reference for the performance evaluation of transfer lines through the use of the decomposition technique. There was, however, a need for improving the applicability of the technique to real systems. Matta and Tolio (1998) extended the decomposition to the case of transfer lines consisting of machines that can fail in multiple modes. Further extensions of the decomposition approach have been driven by the need of widening the set of system architectures that can be analysed. Therefore assembly/disassembly systems have been considered in Gershwin and Burman (2000) and Chiang et al. (2000). General systems, characterised by non-linear flow of material, were analysed in Gopalan and Kumar (1995), Helber (1999), Diamantidis et al. (2004), Li and Huang (2005). Systems presenting closed loop architectures have been considered in Commault et al. (1996) and Gershwin and Werner (2003). The analytical solution for multi-product systems have been presented in Shiue and Altiok (1993), Colledani et al. (2008, 2005b). Recently, new techniques for the evaluation of generally complex system layouts have been developed in $\mathrm{Li}$ (2003), where the "overlapping decomposition" method is proposed, and in Colledani and Tolio (2005) where the "two-level decomposition" is described.

Despite the considerable effort spent in this research field, only a limited number of case studies have been published. Their aim was mainly to provide practical validation of the models, rather than supporting the company in the challenging issue of constantly increasing the productivity of its manufacturing operations. Only a few collaboration projects among industry and academy where these techniques are applied for manufacturing system performance evaluation and improvement can be found in recent literature. Burman et al. (1998) focuses on the application of analytical methods to design a system for manufacturing ink-jet printers at Hewlett-Packard Corporation. The authors showed great benefits for the company through the addition of buffer space in the manufacturing line supported by the use of approximate analytical techniques. Almgren (2000) is based on the experience of Volvo Car Corporation. The author examined the pilot 


\section{ptember 26,2008 \\ nal of Production Research \\ International Journal of Production Research}

production and the manufacturing process start-up of the Volvo S80 model. The goal of this case study was to contribute to the understanding of the way the ramp-up process was affected by certain types of disturbances. Liberopoulos and Tsarouhas (2002) deals with a project to determine cost-efficient ways of speeding up the croissant processing lines of Chipita International Inc., one of largest Greek manufacturers of bakery products and snacks. In this case, the installation of a properly sized in-process buffer at a specific point of the line led to a reduction of the failure impact and an increase of the system efficiency. Patchong et al. (2003) relates to an improvement study of the car body production at PSA Peugeot Citroën, where an iterative three-step design method was developed to improve the system throughput with the minimal capital investment and no compromise in terms of quality. Alden et al. (2006) reports the methodologies and the results achieved by General Motors Corporation in a long-term project to predict and improve the throughput performance of its production lines. Freiheit et al. (2007) examines the investment and operational cost differences between high-volume serial CNC-based machining lines and parallel CNC-based machining lines. Five key tasks to obtain the cost analysis for a given line configuration are proposed.

Nowadays technological advances are increasing the possibilities of applying knowledge based techniques for performance evaluation and improvement. Indeed, manufacturing plants are more and more frequently equipped with systems for automatic data collection, where a big amount of production data is stored and available for further analysis (Colledani et al. 2007). In spite of the cost of implementation and maintenance of such systems, the information collected is still used only for obtaining aggregated and static performance measures. The use of data for deriving knowledge on the system dynamic behaviour, which is the basis for improving the system performance, is far from being achieved. As a matter of fact, companies rarely use approximate analytical tools on their own and seem to prefer simpler trial and error procedures and experience based approaches (Nyhuis et al. 2005).

This paper presents the results of a collaboration project among Politecnico di Milano (Milano, Italy), Kungliga Tekniska högskolan (Stockholm, Sweden) and Scania CV AB (Södertälje, Sweden) in the area of manufacturing system productivity improvement. The project enabled, with respect to the 6 -cylinder engineblock machining line, high increment in throughput and considerable savings in work in progress (WIP); it also gave the company an integrated and formalized methodology for manufacturing system configuration, reconfiguration and continuous improvement. Such an approach can be used several times during the system life-cycle, i.e. every time there is a change in the market requirements, a change in the product features, or company organizational changes take place and drive toward a manufacturing system modification.

The scope of this paper is twofold. Firstly, it proposes a new framework which integrates the performance evaluation method into a broader procedure for manufacturing line productivity improvement. The approach grounds on real production data directly collected from the workshop through the production monitoring system. Secondly, it proposes the application of approximate analytical methods to the dynamic behaviour analysis and the performance evaluation of a real production line. The aim is to highlight the benefits deriving from the use of these tools in companies and to provide a set of guidelines that should be considered for practical use. 


\section{Scania's Production Context}

Scania is a leading worldwide manufacturer of heavy trucks and buses, as well as industrial and marine diesel engines. The group employs more than 35.000 people in 100 countries and in 2007 achieved net sales for 14.2 billion USD.

The considered production system (called D12 line) belongs to the Engine Melt and Machining unit placed in Södertälje (Sweden) and machines 6-cylinder engine-blocks starting from casted blocks. In 2006-2007, when the analysis was carried out, the line consisted of 23 stations (CNC machines, robots for light assembly, washing equipment and a final manual quality control station), performing 23 main groups of operations, connected by 22 intermediate storage buffers (conveyors). After the entire process on the D12 line, the blocks are ready for the assembly operations in another line. The material flow between the lines is controlled by a kanban system. The layout of D12 line is shown in Figure 1 . The line has two curves where two long buffers with relevant capacity are installed. The use of these long conveyors is not only motivated by layout reasons; they also allow to carry out maintenance actions in a portion of the line without stopping the entire line.

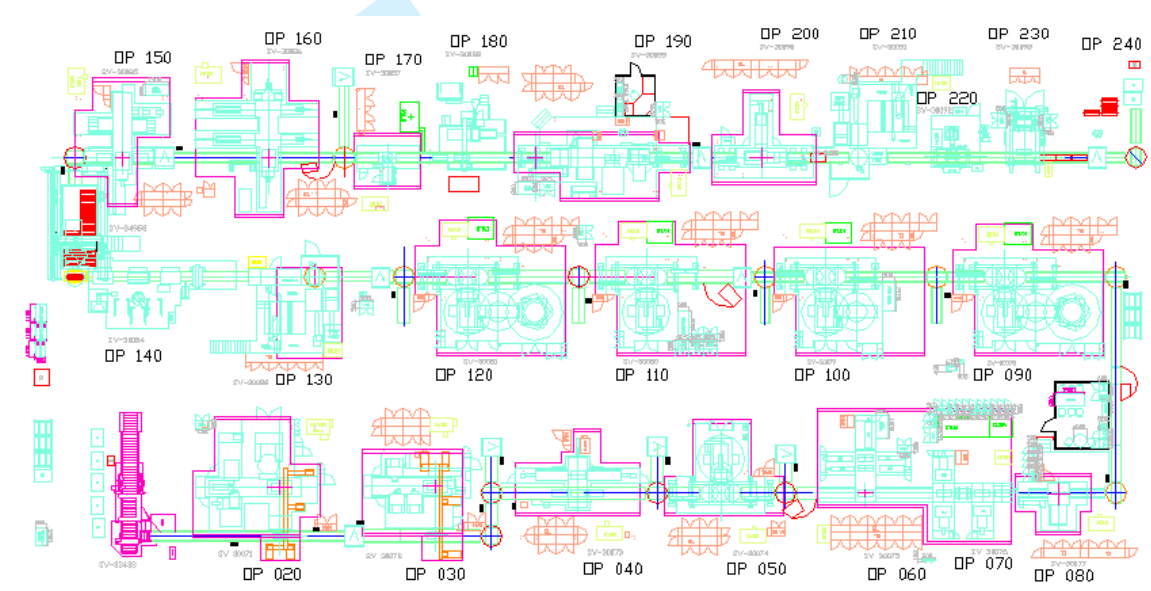

Figure 1. Layout of D12 engine-block machining line in 2006-2007, when this project was run. Parts enter the system at station OP 020 (bottom left) and exit at operation OP 240 (top right).

Each station is affected by 6 different failure modes on average, such as tool breakage, feeder damage or other disruptions regarding mechanical and electronic devices. Each failure mode is characterized by its own occurrence frequency and repair time, depending on the proper failure cause and the operation it belongs to. As an example of multiple failure causes, operation 090 stops on average every 8 hours due to a tool breakage and every 10 hours due to an electronic failure. Operation 040 stops for the same causes in average every 21 and 84 hours, respectively. The total number of failures affecting the line amounted to 147.

Most of the manufacturing systems at Scania are equipped with an automatic data collection system, called PUS (from the Swedish term ProcessUppföljningsSystem). Figure 2 shows a scheme of the PUS system. Different data, such as the duration of down, operational and idle states, are logged accurately for each station by the central PLC (Programmable Logic Controller). Data are stored in the company database, where they are accessible through SQL (Structured Query Language) queries. The PUS system was developed starting from 1998 by B4Industry company in close collaboration with Scania (the PUS system has been recently taken over by Prevas company). In spite of the wide range of production data that 


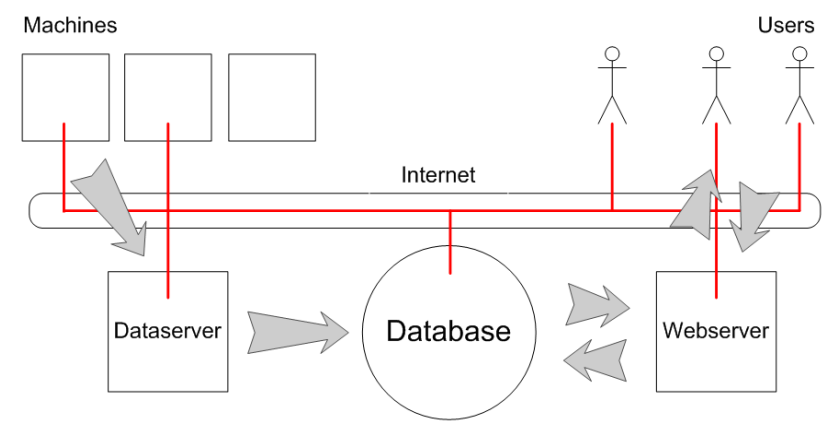

Figure 2. General scheme of the automatic data collection system that equips most of Scania's manufacturing systems.

the system allows to collect, only basic performance indicators are calculated, such as average availability of each resource, number of parts produced in a shift and number of parts to be processed before a tool change. The large amount of data regarding machine reliability remains stored in the company database without being transferred into knowledge for supporting the decision making process.

The analysis focused on the D12 line because its performances were particularly critical for the company. Its production rate was in fact $19 \%$ lower than expected and the line frequently caused starvation of the downstream assembly line, visible through the accumulation of kanbans at the end of the line.

\section{A New Approach to Continuous Improvement}

Continuous improvement is very important for Scania and especially for the D12 line, in which the high level of strain makes kanbans pile up at the end of the line. The efficiency of the line is assured by operators that discuss after every shift defining strategies to solve the main problems encountered during the shift. Scania staff's focus is on machine efficiencies and in particular on the reduction of failure repair times. Simplicity of resolution generally gives the priority in case of multiple breakdowns, that is disruptions that are easier to repair are fixed first. A different approach to continuous improvement was suggested during the project, built and used in order to:

(1) have a deeper understanding of the system behaviour;

(2) capture the main interactions among the system variables;

(3) analytically identify the main causes affecting the system performance;

(4) define suitable actions characterized by the most positive impact on the performance;

(5) check the effect of the system modifications before the implementation in the real world.

An overview of the proposed methodology is synthesized in Figure 3: the four main steps are presented in the boxes, while the tools used to carry on these phases are indicated close to the arrows.

The procedure starts with the Real System Observation. In this phase, the system layout, the part flow management rules, and the characteristics of the equipment composing the system are observed in order to derive the basic features of the model to be adopted in the analysis. The data collected by the Monitoring System are stored in the central database. They are statistically treated, with the aim of characterizing the reliability of the resources composing the system and to obtain the parameters modeling the failure and repair phenomena (Data Collection 
Figure 3. Proposed framework for manufacturing system analysis and continuous improvement.

83 Analysis). The most appropriate production System Model to be used in the analysis is then selected. The first criterion which drives the selection of the proper model is the specific feature of the part flow mechanism in the system. Discrete models are generally used to model synchronous production systems; all machines start producing a workpiece at the same time instant if they are operational and not impeded by the other stations in the line. The flow of parts through the stations composing the system is modeled as discrete. Continuous models are generally used to model asynchronous production systems; each machine can have different processing rates and the flow of parts in each buffer is similar to that of a fluid in a tank controlled by two valves. For a discussion on the use of these models see Gershwin (1994). The second criterion concerns the system layout. Different system architectures can be considered in the model such as serial lines, loop systems, split-merge systems or lines with rework, among others. The third criterion regards the failure and repair mechanism. According to the number of different disruption types affecting the availability of each resource composing the system a single failure mode or a multiple failure mode model can be taken into consideration. Once a production system model is selected, the proper analytical methodology allows evaluating the main performance of the system (Performance Evaluation), enabling a deep understanding of the system dynamic behaviour. Finally the Sensitivity Analysis $\mathbb{E}$ System Optimization are used to define the best improvement actions, considering both the impact on the performance and the implementation cost (Performance Improvement). Once the best improvement actions are defined, they can be implemented in the real system, closing the improvement loop (Improvement Action Implementation).

Such framework aims at formalizing the continuous improvement phase, supporting the decision making process and giving the analytical justification to the implemented actions. It is designed to be run several times during the system life-cycle, ensuring its competitiveness by adapting the system performance to the production requirements. Each time it is applied to the real system, it provides in output the practical guidelines to obtain performance improvements, estimating the impact of particular actions against others. The use of analytical methods makes the approach especially suitable in those cases in which several performance improvement actions are possible, with a different impact that is not quantitatively known a priori. Moreover, it represents a formal procedure to drive and push the search for improvement actions through the use of knowledge based tools also in those manufacturing contexts in which well known and eradicated procedures are applied. In the next sections, a stepwise description of the methodology is proposed, along with the numerical results of its application at Scania. 


\section{Detailed Description of the Methodology}

\subsection{Monitoring System}

The monitoring system is a critical tool because it represents the direct link between the methodology used for the analysis and the real system. The accuracy and the completeness of the collected data strongly affect the quality of the succeeding analysis. Figure 4 represents the structure of the system implemented in D12 Line. Machines are connected to the monitoring system through PLC for collecting state changes into the PUS database. This allows to trace the machine history in terms

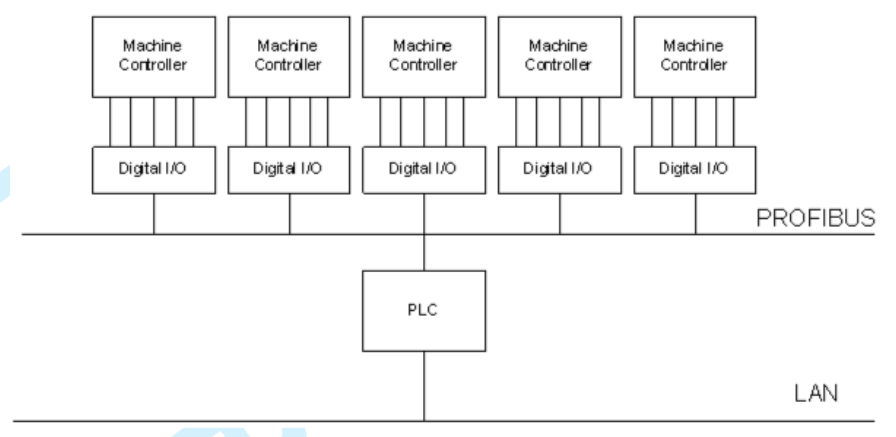

Figure 4. Representation of the monitoring system of D12 line.

of operational, down and waiting states. In Figure 5 a small portion of the real sequence of these states is reported for the first machine of the line ("MachineID" 289). It represents the output of a query on the company database and shows, for each state in which the machine has been, the relative type ("OperationalStateTypeID"), the failure mode ("DisruptionTypeID") in the case of down state, the occurrence date ("Date") and time ("Time") and the duration ("sDuration"). Through these data different sets of important information about the machine's behaviour can be derived, such as the portion of time the machine was operational, the average duration of a certain failure or the average time between the occurrence of two failures of the same type.

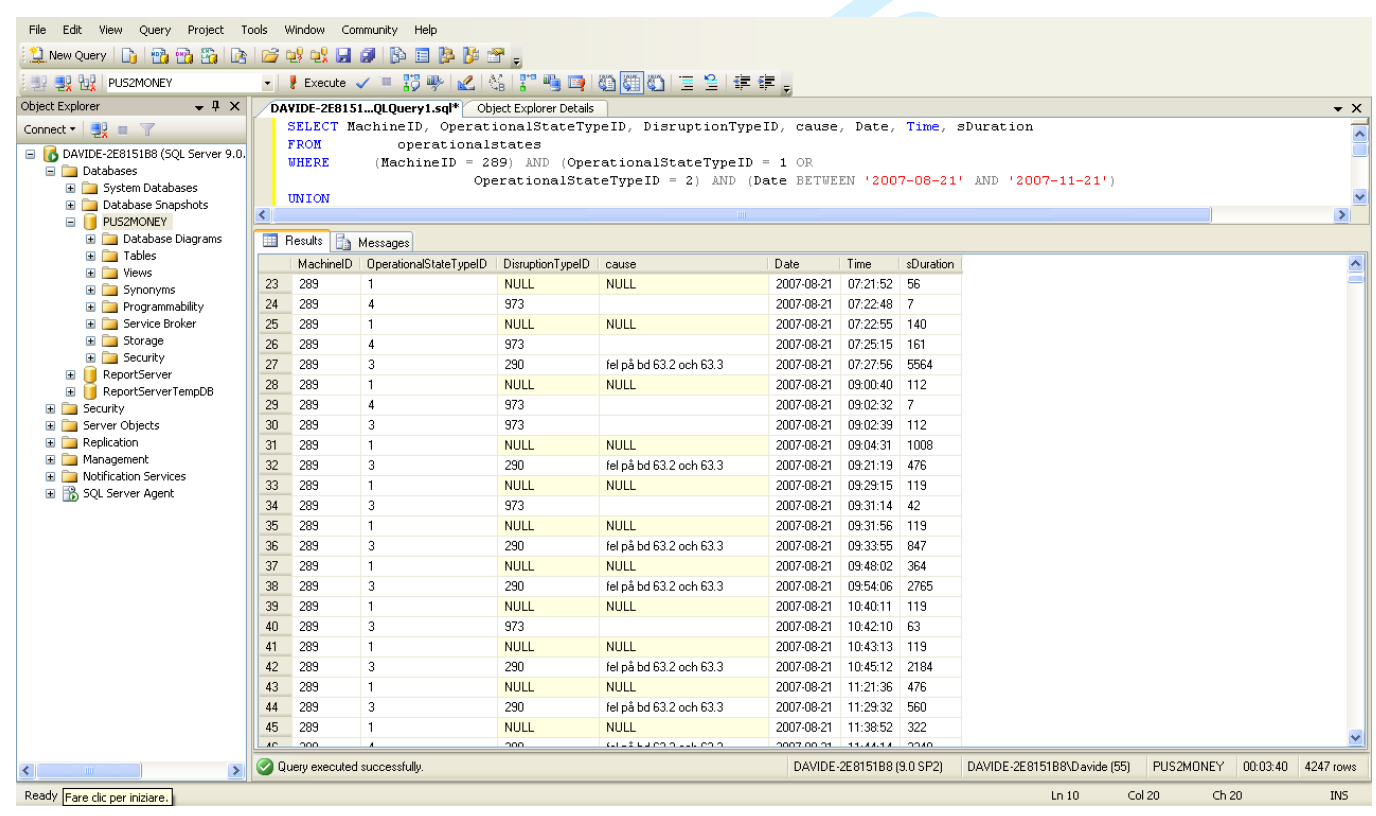

Figure 5. Output of a query on the company database reporting a small portion of the real sequence of states for the first machine of D12 line. 


\subsection{Data Collection \& Analysis}

The goal of this phase is to obtain data concerning machine cycle times, buffer capacities and production disturbances directly from the workshop. In particular, the reliability parameters are derived from the production data in terms of Mean Time To Failure (MTTF), that is the mean operative time between the end of the repairing of a failure and the occurrence of a new failure of the same type, and Mean Time To Repair (MTTR), that is the mean time from the occurrence of the failure and the end of the repairing process. A sample of production data between September the $1^{\text {st }} 2005$ and April the $30^{\text {th }} 2006$ has been collected as described in Section 4.1, considering all the machine states (working, idle or failed) logged by the monitoring system along with the relative duration. Starting from this information, all the Time to Failure (TTF) and the Time To Repair (TTR) are calculated for each machine and disruption type.

Figure 6 shows, for instance, the frequency histogram representing the empirical distribution of the Time to Failure (TTF) of the "tool breakage" disruption for operation 020 . In order to obtain this distribution the 279 observations of the failure phenomenon registered in the database over the observed system life time are considered. The respective MTTF is computed as the mean of all these values. This procedure is automatically repeated for all the 147 failure modes registered in the database which stores the data collected by the production monitoring system. The calculated failure parameters represent a first important information for the

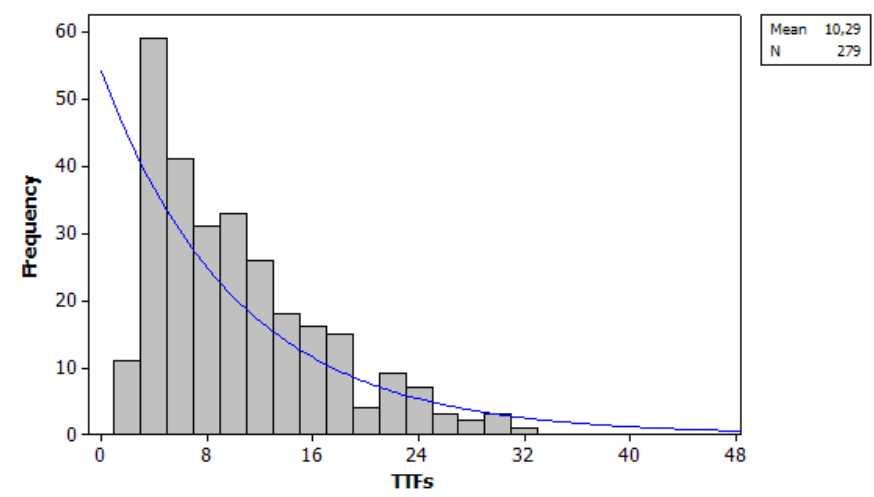

Figure 6. Bar chart with exponential fit for all time to repairs of the "tool breakage" failure mode of operation 020 .

company because they highlight how often, in average terms, a particular failure occurs and how much an operator takes to recover the machine from it. For instance, Figure 7(a) charts the MTTF and the MTTR of all the occurring failure modes for machine 020: different disruption types have different occurrence frequencies and repair times due to the specific nature of the failure and the repair action required. Figure 7(b) represents the same parameter for the single "tool breakage" failure for all the machines in the line: it can be noticed that the mean repair times are very similar while the mean times to failure are highly different among the machines. Similar graphs are derived for all the failure modes affecting the production of the other machines in the line. Such results were discussed with the production manager and the D12 line manager in Scania, with the objective of providing a first empirical validation of the input data for the approximate analytical method based improvement procedure.

A similar approach has been adopted to derive the information concerning the machine cycle times. The cycle time is defined as the time each station requires for processing one block. By combining the information concerning the time instants 


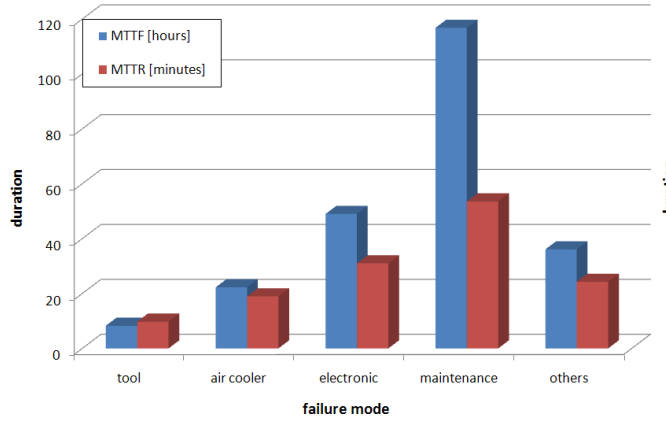

(a)

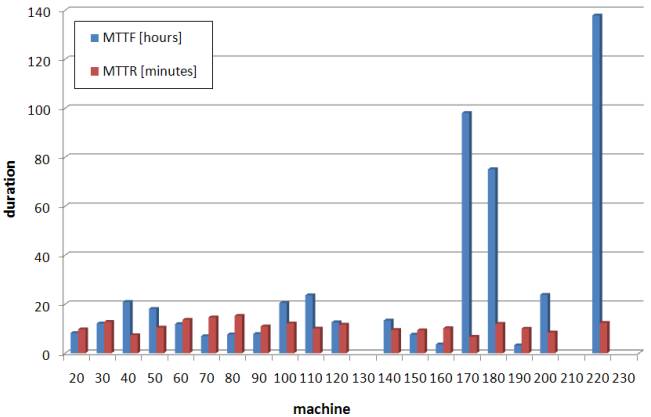

(b)

Figure 7. MTTF and MTTR of the different failure modes of machine 020 (a) and MTTF and MTTR of the failure mode "tool breakage" for each machine of the line (b).

in which a block enters and leaves each station, available in the database, it was possible to obtain a sample of cycle times detected during the system observation period, for each machine. As the machines composing the system are fully automatic (CNC machining centres and robots), the variability of the cycle times observed in a sample was very small. Machine cycle times are reported in Figure 8(a). As it can be noticed, cycle times of different stations are almost equal, thus the flow line is fairly balanced (the coefficient of variation of the cycle times of the machines in the system is 0.102$)$. Finally, according to the system layout, the capacity of the buffers in the line can be obtained (Figure 8(b)).

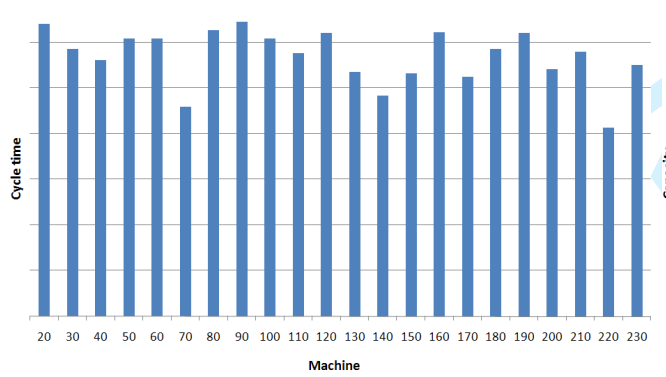

(a)

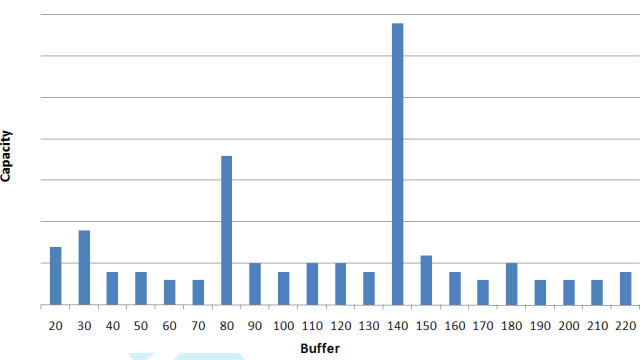

(b)

Figure 8. Machines cycle time derived from the production monitoring system database and capacities of the inter-operational buffers. Numerical values are omitted for confidentiality reasons.

\subsection{System Model}

This phase involves the selection of the proper system model enabling to describe the dynamic of the material flow in the real system through a set of realistic assumptions. The first aspect that is taken into consideration is the part flow mechanism. According to the analysis carried out in the previous phases, the main features of the material transfer among the production stages are analysed. Machine cycle times are almost deterministic and they only slightly differ from one machine to the other. The line is therefore almost synchronous. The material flow is intrinsically discrete and the capacities of buffers linking the machines in the system are fairly small. In literature it is shown that while modeling a line characterized by discrete material flow with a continuous fluid model, accuracy problems may be observed (Gershwin 1987), especially when the buffers in the line are small. Moreover, Koster and Wijngaard (1989) shown that when cycle times are of the same order of mag- 
nitude as repair times, the continuous model loses accuracy. Since the D12 Line presents similar characteristics, a discrete material flow model is selected. However in order to model in detail the slightly different processing times of machines, the system time unit is set to the fastest machine processing time and an additional failure mode is added to the other machines, reducing their operational time.

The second relevant aspect which drives the decision about the production system model is the system layout. In the analysed line, machines and buffers are located to form a S-shaped layout (Figure 1). However, following the sequence of operations, the line layout can be "stretched" and modeled as a linear layout. Raw materials enter the line in the first station, Operation 020 named in the model $M_{1}$, go through all the 22 stages in the line and are released as final products downstream the last machine of the line, Operation 230 named $M_{22}$ in the model. The last operation 240 involving only a manual $100 \%$ quality control is not considered in the model. Since the time required for this operation is approximately $1 / 4$ of the cycle time of the other stations, it never disturbs the production of the upstream portion of line. No reprocessing of parts is possible in the system. Moreover, parts are not subject to alternative paths in the system and parallel system branches are not present. The amount of material entering the line is regulated by the kanban production control rule. However, according to the production data considered in the period of observation of the line, the amount of kanbans activating the production of the D12 line was always greater than one, causing the line to be operational also during the weekends. Therefore, in accord with the line manager, continuous supply of parts in front of the first machine of the system has been modeled. Finally, the only first in-first out rule is active at each production stage. The selected production line layout adopted to model the real system is represented in the upper side of Figure 9, where machines are represented as squares and buffers are represented as circles.

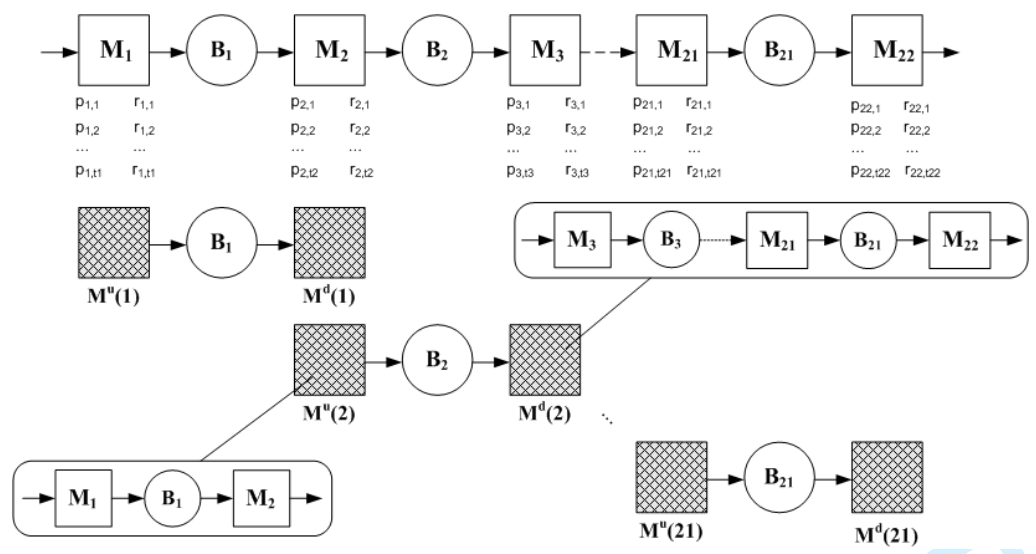

Figure 9. Decomposition principle. Machines are represented by the squares, while inter-operational buffers by the circles. $p_{k, i}$ and $r_{k, i}$ represent respectively the failure and repair probability of disruption mode $i$ of machine $k$.

The last aspect to be considered for the proper production system model selection is the failure-repair mechanism of the machines composing the system. Since the machines in the real system are affected by several types of disruptions, which are characterized by highly different failure and repair parameters, the selected production system model includes the presence of multiple failure mode machines. In multiple failure mode models each disruption type affecting the several machines is directly considered, therefore failure-specific MTTF and MTTR must be evaluated. This feature of the model allows for deeper detail in the failure repair 


\section{ptember 26,2008

mechanism, reducing the approximation of the real system model. It is important to underline the relevance of choosing a model so it is able to treat different failure modes. From one side, it provides much richer information about the system behaviour than a model based on an aggregate failure mode, allowing the evaluation of the impact of each single disruption on the overall system performance. This is useful for isolating the root causes for the system loss of performance. In addition, it ensures more reliable estimations of the performance measures. Indeed, since the machines are affected by different disruption types with extremely different failure and repair probabilities, to use an aggregate failure model means to average the MTTF and MTTR of all the failures of a particular machine in a unique fictitious disruption. This simplification captures the average impact of the failure on the machine efficiency, but fails in modeling the variance of the machine down times, overestimating the performance of the system (see Gershwin et al. 2002, for detailed comparisons between the two models).

According to the previous considerations, the detailed list of assumptions adopted to model the production system dynamic behaviour follows:

- The modeled flow production line is composed of 22 unreliable tandem machines separated by 21 dedicated buffers with limited capacity. The $k^{\text {th }}$ machine and dedicated downstream buffer are denoted with $M_{k}$ and $B_{k}$ respectively: $B_{k}$ has capacity equal to $N_{k}$ and it contains only pieces already worked by $M_{k}$.

- A generic $M_{k}$ is blocked if the downstream $B_{k}$ is full. A generic $M_{k}$ is starved if the upstream dedicated buffer $B_{k-1}$ is empty.

- The first machine $M_{1}$ is never starved and the last one $M_{22}$ is never blocked. This means there is always availability of raw parts waiting in front of the first machine in the line and there is always space available for storing finished products downstream the last machine in the line.

- Time is discrete and all the machines have equal and constant processing times. Time is scaled so that processing cycle of each machine takes one time unit (the real time interval modeled as the time unit is omitted for confidentiality reasons). Machines start their operations at the same time period.

- Transportation time among buffers and machines takes negligible time compared to machining operation time.

- Machine $M_{k}$ is unreliable and it is prone to fail in $t_{k}$ different modes.

- Whenever a machine $M_{k}$ begins to process a workpiece, there is a constant probability $p_{k, i}=1 / M T T F_{k, i}$ that it fails in mode $i$.

- Whenever a machine $M_{k}$ is down in mode $i$, there is a constant probability $r_{k, i}=1 / M T T R_{k, i}$ that it is repaired from failure of mode $i$.

- For each machine $M_{k}$ and each failure mode $i$, times to failure $\left(T T F_{k, i}\right)$ and times to repair $\left(T T R_{k, i}\right)$ are considered to be geometrically distributed with mean $M T T F_{k, i}$ and $M T T R_{k, i}$.

- Failures are operation dependent (ODF), a machine that is not processing a workpiece (i.e. which is starved or blocked) cannot fail.

- Workpieces are not destroyed, rejected or added at any stage in the line.

- Dispatching rule: all machines take pieces from the upstream buffer according with the FCFS (First Come First Served) rule.

- Blocking before service is considered (Gershwin 1994).

This set of assumptions completely defines the considered production system model and its dynamic behaviour. The suitability of these assumptions to model the real system under analysis has been checked through a statistical analysis of the data collected from the database. In particular, for each failure mode, the geometric distribution used to model the failure-repair mechanism has been verified through 
tests of hypotheses applied to the empirical distributions similar to that of Figure 6.

\subsection{Performance Evaluation}

According to the selected production system model, the approximate analytical method described in Matta and Tolio (1998) for studying discrete transfer lines with multiple failure mode machines is used to calculate the main performance measures of the system. The method is based on the line decomposition approach (Figure 9). In particular, the line is decomposed into several two-machine onebuffer subsystems, also named Building Blocks. Building blocks are easy to analyse in an exact way because of their lower complexity compared to that of the original system. They are solved with the exact analytical method described in Gershwin et al. (2002), which is based on the Markovian analysis of the system. Machines in the building blocks are named pseudo-machines since they mimic the behaviour of the entire portion of real line upstream or downstream the considered buffer. The coherence among building blocks is made possible by the definition of decomposition equations that establish proper relationships among them. Parameters of the pseudo-machines have to be defined so that each building block represents the behaviour of the original system in order to respect the equivalence between the flows passing through the buffers of the original line, and through the buffers in the building blocks. The parameters to set are the values of the variables assigned to each building block that rule the interruption of the flow of parts (failure and repair probabilities of the pseudo-machines) in each buffer. These are calculated by solving the decomposition equations following the so-called DDX algorithm (Dallery et al. 1988), which in turn considers all the upstream pseudo-machines and the downstream pseudo-machines in the building blocks, until convergence.

Approximate analytical models have been chosen because they effectively support the proposed performance improvement framework thanks to their quickness and accuracy in evaluating the performance of manufacturing systems. Moreover, they provide a useful guideline for practitioners in selecting those system parameters that are needed to capture the most important aspects of the line dynamic behaviour; as a result they only need a few input parameters. Furthermore, they are general because they can be applied to most of the Scania's manufacturing lines, since they share similar characteristics.

The approximate analytical method used in the analysis of the real system is implemented in a software tool and can therefore be used like a "black box", by both operators and production engineers. In particular the software used in the project is named FlowLine (Figure 10) and was developed at the Department of Mechanical Engineering of Politecnico di Milano. The background of Figure 10 shows the layout of the original line as a set of machines (squares) and buffers (circles). The software input parameters are the probabilities of failure and repair for every machine and failure mode, as well as the machines' cycle time and interoperational buffer capacities. An example of the outputs the software provides is shown in Figure 10. The pie chart in this example displays the percentage of time, computed after the evaluation, operation 090 spent in operative (blue color), failed (white color), starved (green color) and blocked (red color) states.

The complete list of performance measures Flow Line provides in output follows:

- machine efficiencies in isolation;

- average machine state durations;

- average production rate or throughput;

- average buffer levels; 
Figure 10. Overview of the software Flow Line.

- average work in progress;

- average lead time;

- machine starvation and blocking probabilities along with the related root causes.

\subsubsection{Efficiency in Isolation}

The efficiency in isolation is defined as the production rate of one machine if it were a stand alone machine, i.e. not idle or blocked by any other machine in the system. This measure is useful to detect bottlenecks of the line, i.e. those machines which regulate the behaviour of the whole line having higher impact on the overall system throughput. The values of the efficiency in isolation calculated for the D12 line in Scania are showed in Figure 11(a) and can be obtained through the following Equation (Gershwin et al. 2002):

$$
e_{k}=\frac{1}{1+\sum_{i=1}^{t_{k}} \frac{p_{k, i}}{r_{k, i}}}
$$

where the index $k=1, . ., 22$ refers to the generic machine $M_{k}$ in the system and $i=1, . ., t_{k}$ refers to the specific failure mode of this machine. The smallest efficiency in isolation value represents the maximum throughput obtainable by the line in the ideal case of infinite buffers among machines, i.e. if the machines in the line were completely decoupled. Indeed, in this case there is no interference among different stations and the line produces at the rate of its lowest efficiency machine. The value of the maximum throughput in the ideal case of infinite buffer can be obtained through Equation (2). Figure 11(b) represents the infinite buffer level 
On the contrary, if no buffer place is installed among machines, a failure of a particular machine immediately idles all the other downstream stations and blocks all the upstream stations. In this case, the obtainable throughput reduces, especially for a long line such as the D12 line. In this situation, under assumption of operation dependent failures, a good approximation of the efficiency of the line is given in Buzacott (1967):

$$
T H(0)=\frac{1}{1+\sum_{i=1}^{T} \frac{p_{i}}{r_{i}}}
$$

where $T$ indicates the total failure modes in the line. The current and the target throughput of the line are therefore included between these two bounding values.

\subsubsection{Average Throughput (TH)}

The throughput of a production system is defined as the number of parts produced by the system in a time unit. Figure 11(b) shows the behaviour of the throughput curve on the total buffer capacity for the analysed system. This curve has been obtained by allocating the different values of the total buffer capacity (x-axis) in the system, in an optimal way. As it can be noticed, the curve tends to the efficiency in isolation of the smallest efficiency in isolation machine (operation 090) as the total allocated buffer capacity tends to infinity. Moreover, it tends to the value obtained in Equation 3 as the total buffer capacity tends to zero.

The value of the throughput provided by an approximate analytical method is almost always compared in literature with the throughput provided by an exact model (in general a simulation model), built with the same assumptions of the approximate analytical method whose accuracy has to be tested. The logic which is behind this approach is to test the approximation introduced by the decomposition technique with respect to an exact technique. Dealing with the problem of improving the performance of a real system, in this paper also the validity of the assumptions introduced in the production system model must be tested. In case the method provides accurate performance estimation but its assumptions do not apply to the real system, the estimated performance is far from the real one and 
the following analysis is unreliable. Therefore, being the real system data available in the production monitoring system database, this information is used to test the validity of the performance evaluation phase. The comparison between the value estimated by the approximate analytical method and the one calculated through a sample of representative historical data is performed. In particular, a significant sample of daily production data, drawn in the same period considered for the calculation of TTFs and TTRs, is extracted from the database. After having checked the normality distribution for these values, the bilateral $95 \%$ confidence interval on the mean is computed. Figure 12 shows that the error in the estimation of the average throughput is $4 \%$. More interesting, the value is included in the confidence interval on the mean for the normal distribution ( $42 \% \mathrm{P}$-value) of the analysed sample of data. This analysis provides a validation of the method, in terms of ac-

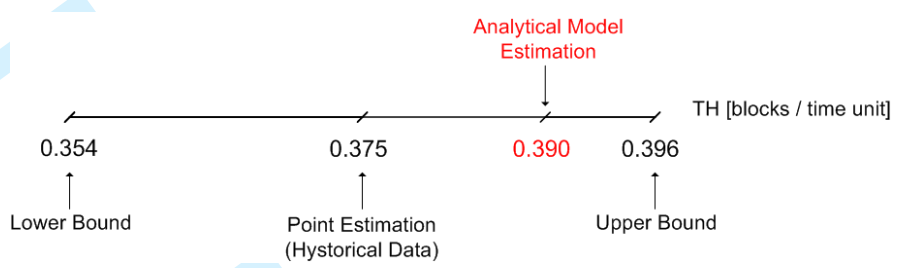

Figure 12. Throughput estimation and bilateral 95\% confidence interval on the mean for a normal distribution with unknown variance.

curacy, for the current configuration of the system. Since the method grounds on a detailed description of the system dynamics, this information ensures that the method correctly captures the main phenomena determining the dynamic of the material flow in the line. Therefore, it can be used for driving future analysis with a high level of confidence in the provided results.

\subsubsection{Average Buffer Level (BL)}

Besides the throughput, another important manufacturing system performance indicator is the average system Work in Progress (WIP) that refers to the number of parts that are present, in average terms, in the system. As it was highlighted in the section 4.4.1, the inclusion of buffers between machines allow increasing the average throughput, by partially de-coupling adjacent stations. On the contrary, the more buffer places are installed in the line the more WIP is present between the stations. Besides the investment and operational costs of buffer modules and the opportunity cost due to floor space occupation, engine blocks are high value parts, therefore their cost in terms of circulating capital is relevant. The level of WIP is calculated through the estimation of the average level of each buffer in the line. Figure 13 shows the average buffer level calculated for every inter-operational buffer place. Once the average level of each buffer in the line is known, the total WIP can be calculated with the following formula:

$$
W I P=22 \cdot T H+\sum_{k=1}^{22} B L_{k}
$$

where the first term is related to the number of parts that are on average under process in the 22 machines and the second term refers to the number of parts that are on average stored in the buffers.

\subsubsection{Average Lead Time (LT)}

The average lead time is the average amount of time one block spends in the system, i.e. between the instant in which the casted block enters the line and the 
instant in which it finishes the last operation. It is related to the average amount of inventory and can be calculated through Little's law:

$$
L T=\frac{W I P}{T H}=191.75[\text { time unit }]
$$

By comparing such value with the sum of all the machine cycle times it is possible to show that the entire process adds value only during the $15.82 \%$ of the time a block spends in the system (Added Value Time \%); the remaining fraction of time represents idle periods the blocks spend in the queues between the machines, waiting to be processed. This value can be obtained by using the following equation:

$$
\text { Added Value Time } \%=\frac{\sum_{k=1}^{22} C T_{k}}{L T}=15.82 \%
$$

Where $C T_{k}$ represents the cycle time of the $k^{\text {th }}$ machine.

\subsubsection{Starvation and blocking probabilities 8 relative root causes}

When a failure of a specific machine $M_{k}$ occurs and persists long enough, the adjacent upstream buffer $B_{k-1}$ may fill up, forcing its upstream machine $M_{k-1}$ to stop processing parts. Such a forced state of $M_{k-1}$ is called blocking. Similarly, the failure of $M_{k}$ can cause the downstream buffer $B_{k}$ to empty, forcing the downstream machine $M_{k+1}$ to stop processing parts. In this case, the machine $M_{k+1}$ is said to be starved. Blocking phenomenon propagates upstream the line, starting from the failed machine and with a decreasing effect toward the first machine of the line, while starvation phenomenon propagates downstream the line, with a decreasing effect toward the last machine.

The probabilities of these phenomena calculated in the D12 line are charted in Figure 14(a) and 14(b) for each station of the line. They represent the portion of time the machine cannot work because it is idled by other stations. The first consideration suggested by these graphs regards the impact of buffers on the propagation of blocking and starvation. In particular, the interrupted vertical lines drawn in the figures among Operations 080 and 090 and among Operations 140 and 150 refer to the locations of two large buffers where the S-shaped layout makes the curves and inverts the direction of the material flow. The two graphs clearly highlight the decoupling effect of these large buffers. Considering Figure 14(a), the buffer among Operations 080 and 090 acts as a filter which smoothes the propagation of starvation from operation 080 to the downstream portion of line. The same action can be observed on this graph for the long buffer among operations 140 and 150. Similarly, in the graph in Figure 14(b), the long buffers between Operations 080 


\section{tember 26,2008

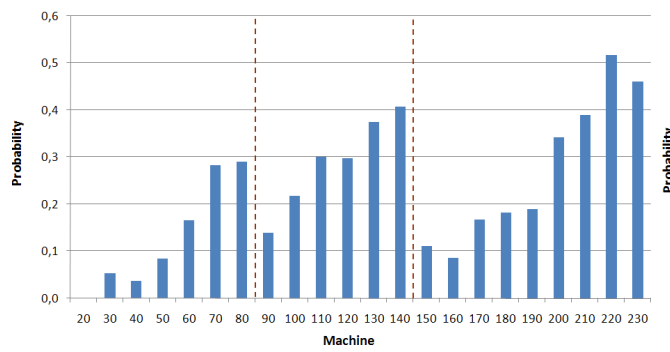

(a)

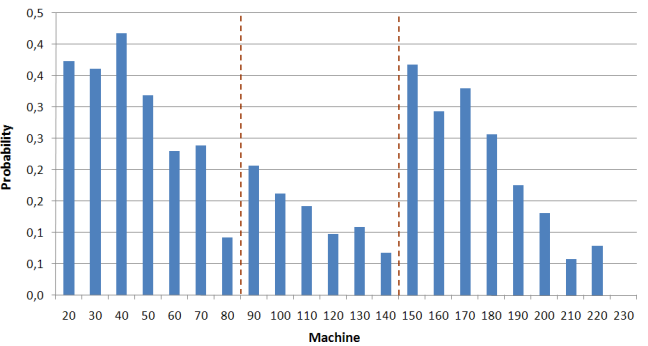

(b)

Figure 14. Machine starvation (a) and blocking (b) probabilities.

and 090 and between Operations 140 and 150 smooth the propagation of blocking to the upstream portions of the line.

The other relevant information provided by these graphs regards the impact of the machine's efficiencies in isolation on the propagation of blocking and starvation. Stations characterized by the relative low efficiency in isolation are critical for the system because they spend a high portion of time in down states, causing the propagation of blocking and starvation to the upstream and downstream machines. In particular, considering the efficiencies in isolation graph reported in Figure 11(a), Operation 090 is the lowest efficiency in isolation machine and Operation 190 is the second lowest efficiency in isolation station. These operations are also named respectively first and second bottlenecks. Focusing on Operation 190, the histogram in Figure 14(a) shows that the starvation probability highly increases moving down the line to Operations 200, 210 and 220, while it is almost constant at the upstream Operations 170 and 180. Regarding the blocking probability (Figure 14(b)) the effect of Operation 190 is less visible being the increment in the blocking probability going from operation 210 upstream the line to Operation 170 practically constant. A similar behaviour can be observed for starvation of Operation 090, since its effect is propagated to the downstream machines until Operation 140. On the contrary, the blocking effect is rapidly mitigated by the long buffer between operations 080 and 090 and therefore only partially propagates to the upstream machines. An important consideration on these graphs regards the impact of blocking and starvation on the bottleneck machines. Since they have the largest effect on the overall system throughput, they should be prevented from being starved and blocked as much as possible. However, in Figure 14(b) it can be noticed that the probability of operation 090 being blocked is relatively high. This provides important insights for the reallocation of the buffer capacity in the line. Additional buffer space should be placed downstream Operation 090 in order to prevent the blocking propagation to this station.

The previous graphs are useful for studying the propagation of blocking and starvation in the line, once they are generated. However, in order to impact on the generation of these phenomena, the search for the principal root causes for blocking and starvation in the line must be performed. Given that every single failure mode is modeled into detail in the approximate analytical method, it is possible to disaggregate starvation and blocking probabilities affecting the performance of each machine $M_{k}$ and link these phenomena to the specific root causes. In particular, the impact of each single failure mode of all the downstream machines $M_{j}$ with $j>k$ on the machine $M_{k}$ is isolated and the portion of the aggregate blocking probability that can be attributed to the failure mode is calculated. In this way, it is possible to identify the specific failures of the downstream machines which have highest responsibility in determining the blocking phenomenon generation, i.e. the root causes for blocking. The same information can be obtained for the starvation 
of each machine $M_{k}$ in the line, by considering the failure modes of each machine $M_{j}$, with $j<k$. While the aggregate information is useful for understanding the dynamic of propagation of blocking and starvation, this detailed information is fundamental to understand the generation of the starvation and blocking phenomena and to reduce their impact. Therefore, the failures that have more critical effect on the line performance should receive higher attention by the maintenance staff.

Figures 15(a) and 15(b) show, respectively, the pie chart and the Pareto analysis for the root causes for blocking of the first working station in D12 line. In this case only the $10.6 \%$ of the causes, that is 15 failure modes out of all the admissible 141 , produce the $80 \%$ of the effects on the blocking of the Operation 020. Performing

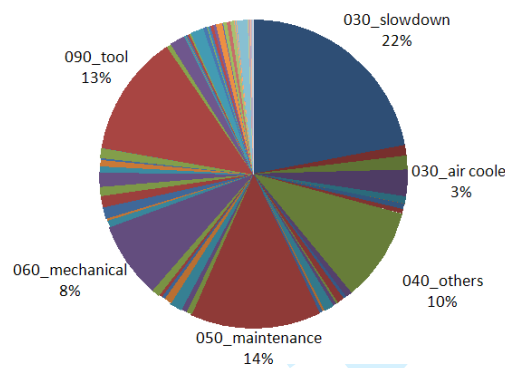

(a)

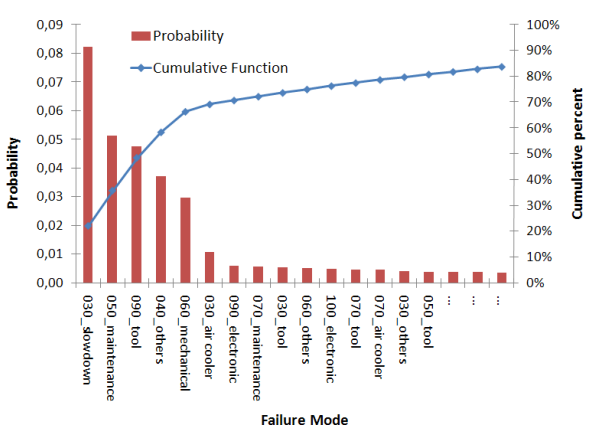

(b)

Figure 15. Pie chart (a) and Pareto analysis (b) for Op 020 - causes for blocking. In the Pareto diagram, for readability reasons, only 18 failures are reported. The other 123 disruptions weight for the lacking $15 \%$.

this analysis for each machine and for both blocking and starvation phenomena, 7 failures out of the admissible 147 have been identified as highly critical in terms of effects on the system throughput. To these failure modes the priority of the repair intervention in case of concurrent disruptions has been assigned by the maintenance staff. Moreover a revised maintenance plan aiming at reducing the failure occurrence for these disruptions has been generated.

\subsection{Sensitivity Analysis and System Optimization}

The previous analysis enables the creation of the appropriate level of knowledge about the system performance and helps in highlighting the critical aspects of the current system configuration. The next step of the procedure selects adequate performance improvement actions based on the developed production system model and the performance evaluation method. The main idea is to model the different improvement actions in terms of changes in the input parameters of the production system model. The evaluation of the performance of the system under the new configuration is then carried out and the impact of the system modification is quantified in terms of performance measures. In this phase, two different approaches are adopted, i.e. Optimization Algorithms and Sensitivity Analysis. The difference among these approaches concerns the way the parameters of the production system model are adjusted to find the improved configuration.

When performing a sensitivity analysis, the interest is focused on the variation of the system performance under small variations of the system parameters. In terms of improvement actions, this means testing the impact of small system modifications that can be easily implemented in the real system without a consistent reconfiguration time and money investment. In this case, the set of new configurations to test in order to find the improvement action to be implemented is generally user guided and knowledge-based. 


\section{eptember 26, 2008

Alternatively, specific optimization problems can be formulated and solved which allow to improve the production system performance through substantial technical or managerial reconfigurations. In this case, once the optimization problem is formulated, the optimization algorithm adopted to solve the problem selects the sequence of new system configurations to be tested. The main drawbacks of these techniques is the fact that normally a large set of system configurations must be tested before reaching the optimal configuration.

In the next section, the results from the application of these approaches to the D12 line in Scania are described. In particular, the sensitivity analysis has been used to investigate the impact of a reduction in the time required to fix the most critical machine failures on the average system throughput. Moreover, in accord with the line manager, two optimization problems are formulated and solved. In particular, an extension of the algorithm proposed in Kuhn (2003) is used to quantify the impact of the number of repairmen on the throughput of the system and to find the best allocation of the available personnel in the line. Finally, the optimization algorithm described in Colledani et al. (2005a) is used to obtain the optimal allocation of the buffer capacity among the machines.

\subsection{Performance Improvement}

Through the approaches described in the previous section three possible improvement actions are analysed:

- Repair Priority Assignment

- Repair Crew Optimization

- Buffer Optimization

The first two approaches are organizational and allow to gain in the production rate with extremely low capital investment. The third one is instead a structural improvement involving a reconfiguration of the line that, in spite of the higher cost, leads to extremely relevant improvement in the performance.

\subsubsection{Repair Priority Assignment}

In the Section 4.4, the identification of those failure types that mainly affect the performance of the line was carried out. By discussing with the D12 line manager and the machine operators in the line, a possible intervention to easily reduce the impact of these disruptions on the loss of performance of the line is identified. It involves the reduction of the time to repair these few critical disruptions, achieved by assigning priority of intervention to them. Indeed, because operators share several machines in the line, a strategy to manage simultaneous failures of machines that privileges high impact failures can lead to shorter repair times of critical disruptions. Moreover, it is not rare that repairing interventions on machines are delayed due to unavailability of spare parts. The priority to these failure types can be achieved by guaranteeing the availability of these parts. Another action for stressing priority is the introduction of alarm systems in order to increase the repairmen reactiveness with regard to these critical failure modes. The assigned priority is modeled through a reduction of the mean time to repair these high impact failures that is estimated at $15 \%$, according to the line manager. The sensitivity analysis is then used to evaluate the impact of this reduction on the performance of the system. The results are reported in Table 1 and show the throughput increment obtained through a $15 \%$ reduction in the MTTR of a single failure mode, selected from the most important ones identified during the Performance Evaluation phase. 


\begin{tabular}{|c|c|}
\hline Failure mode & $\%$ Increase in $\mathrm{TH}$ \\
\hline 180_others & $0.70 \%$ \\
\hline 200_tool & $0.01 \%$ \\
\hline 090_tool & $0.13 \%$ \\
\hline 060_mechanical & $0.22 \%$ \\
\hline 110_others & $0.16 \%$ \\
\hline 120_mechanical & $0.73 \%$ \\
\hline 090_electronic & $0.47 \%$ \\
\hline
\end{tabular}

Such increments are highly significant if compared with the required effort: the action does not involve relevant additional cost or resource allocation in the line, but only the priority of intervention of the available maintenance staff is modified. A $0.7 \%$ increment in the production rate is obtained reducing by $15 \%$ the MTTR of only one failure mode out of the 147 of the whole line. Last but not least, the results of this sensitivity analysis prove the quality of the previous failure identification process, highlighting that the identified failures have a critical impact on the performance of the line.

\subsubsection{Repair Crew Optimization}

In automated flow lines, the impact of machine breakdowns and repairs have a dominant effect on the performance of the systems. As a result, a dedicated repair crew usually exists at the line to clear station jams or machine failures (Stecke and Aron 1985). In the unrealistic case in which one operator per machine is available in the line during each shift, as soon as the failure occurs the repair intervention is started by the operator. However, in order to save operating costs, the number of operators attending the line during the shift is usually consistently lower than the number of stations in the system, that is only a few operators for a line composed by 20 stations is available. As a result, when a failure occurs and all the operators are busy repairing other stations, the machine must wait before the repairing intervention is started. This kind of machine idleness is known in literature as interference (Ferdinand 1971). The interference makes the mean time to repair being generally composed by two terms: the time required for waiting for the operator to be available to attend the failed machine and the time needed to fix the problem. The latter period directly depends on the nature of the disruption, while the former strongly depends on the number and the reactivity of the repairmen allocated to the line. The problem that deals with the interrelations between the system production rate and the operator/workstation interference is known in literature as the machine-repairman or machine-interference problem. For a recent review on methods developed to tackle the machine-interference problem see Haque and Armstrong (2007). The general feature of these approaches is the fact that the repair crew is modeled as a queueing system in which the customers are the machines that need to be attended after a failure and the server capacity depends on the number of available operators. Depending on the assumptions of these methods, different queuing systems are used to model the shared operators.

An interesting approach for practical use is proposed in Kuhn (2003). The paper presents an analytical approach for performance evaluation of an automated flow line with limited repair capacity. The main idea of the method is to couple two queuing systems, one open queueing network consisting of $K-1$ servers with finite buffer capacity, modeling the flow line, and one closed system with finite 
population, representing the repair process, as represented in Figure 16. After the analysis, the repair crew model provides in input to the production system model the updated mean time to repair, obtained considering the machine-operator interference. Similarly, the production system model provides in input to the repair crew model the updated frequency of machine failures, i.e. the expected time between two interventions of the operator, obtained by considering the impact of blocking and starvation phenomena on the machine availability.

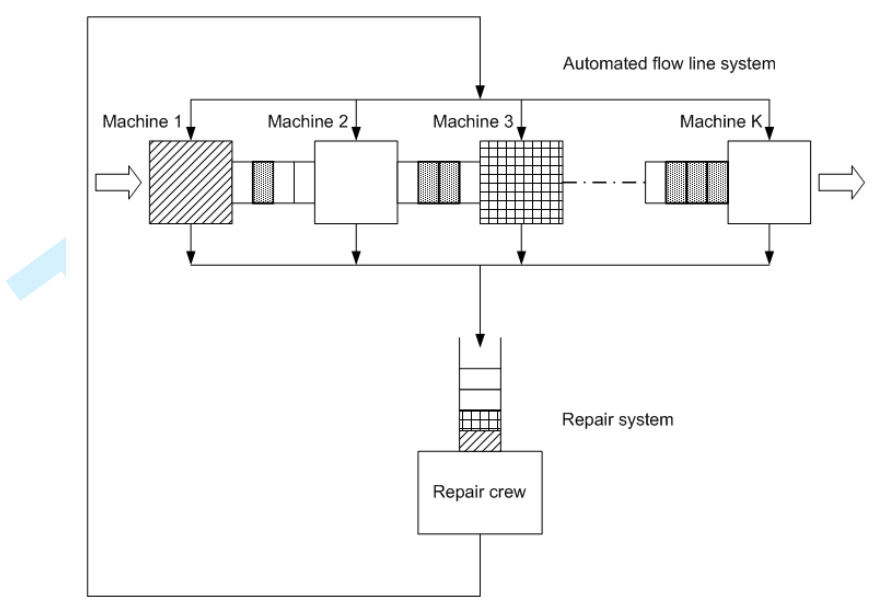

Figure 16. Two-coupled queuing systems representing the automated flow line and the repair crew.

Two different approaches are proposed to coherently solve these queueing models, namely iterative approach and integrative approach. The first approach consists in solving separately the two queueing systems, exchanging the parameters from one system to the other. The method is fairly fast in meeting convergence, but it looses accuracy especially when buffers are small and the repair crew is the bottleneck of the system (Kuhn 2003). The second consists in decomposing the system into several two machine one buffer sub-systems, each one having its own repair system. The performance of each sub-system are then calculated in exact way and decomposition equations are introduced to obtain the overall system performance. The results provided by this method are more accurate than those of the iterative method but the detail modeling of the repair crew-flow line integration is required, thus the mathematical modeling is valid only for the specific introduced assumptions.

In this paper, an extension of the method proposed in Kuhn (2003) is used to analytically solve two different machine-repairman problems in the D12 line. The first problem consists in better allocating among machines the available repairing personnel which attends the line during one shift, considering the different efficiencies of the machine composing the line and the impact of critical failures. The second problem deals with the estimation of the variation in the system production rate due to a different number of operators allocated in the line during one shift. From a methodological point of view, given the complexity of the modeled system, the iterative approach proposed in Kuhn (2003) is adopted. However, the method has been extended to deal with multiple failure mode machines. Therefore, the production line model is evaluated using the decomposition method proposed in Section 4.4 while the repair crew queueing system is solved using the maximum entropy method proposed in Ferdinand (1971).

The identified operators re-allocation scheme is reported in Figure 17. A better allocation of the current number of operators is provided that leads to $2.7 \%$ 
increment in throughput, without any additional allocation of human resources. The solution is identified considering the different machine efficiencies under the constraint of the system layout. The logic is to allocate the operators in such a way that bottleneck machines are repaired as soon as the failure occurs. One operator can be assigned either to a few machines with a low efficiency or to a larger group of machines with a relatively high efficiency. In this way, machine idleness due to failure interference is reduced for those critical machines that have higher impact on the performance of the whole system.

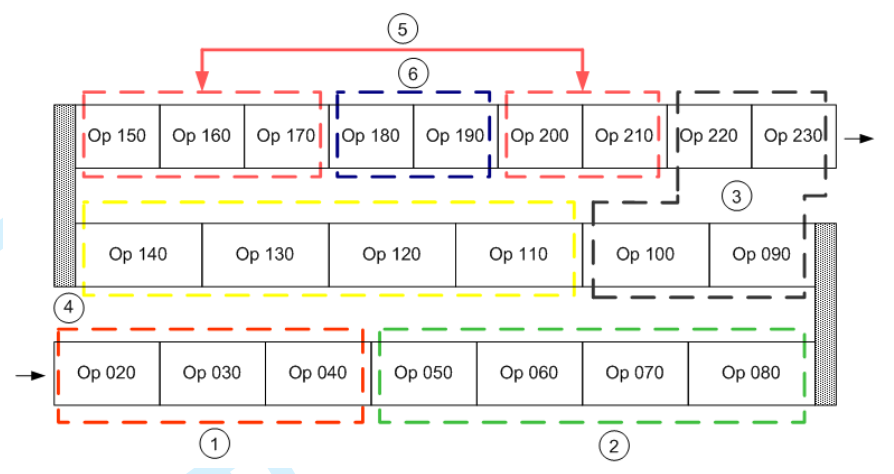

Figure 17. Repair crew re-allocation: best identified solution in the case of six operators.

The solution to the second problem allows charting the throughput of the system as a function of the number of operators assigned to the line, as it is shown in Figure 18. This result is very important for the company because the impact of allocating one more operator to the line is derived. As it can be noticed from the graph, the throughput curve as a function of the number of operators assigned to the line is a monotone increasing concave function. The lower bound is given by the zero operators case. In this unrealistic situation, as long as a machine goes down it never gets repaired, thus the steady state production rate is equal to zero. The upper bound is given by the case in which each machine is attended by one operator, i.e. operators are not shared among machine and no interference is observed. In this

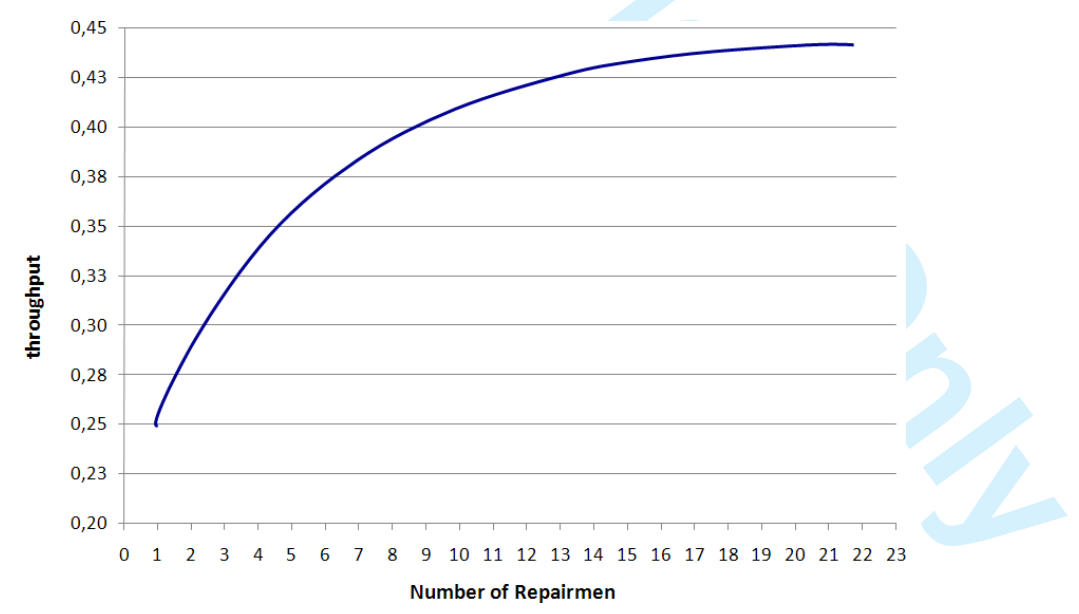

Figure 18. Throughput vs. number of repairmen assigned to the line.

case, the MTTR of the machine is only composed by the portion of time required for physically fixing the problem, i.e. no waiting time is present. In the intermediate cases, a decreasing level of interference of the operators, reading the graph from left to right, increases the system throughput, with smaller impact as the number of operators increases. In particular for the case under analysis, by introducing an 


\section{ptember 26,2008

additional operator (from 6 to 7 operators) to the line during one shift an increment in the throughput of about $5.5 \%$ can be achieved. This modification of the system involves the cost of the additional operator but entails a fairly high increment in the system performance, by giving the operators a higher reactivity to failure occurrence. Finally, a similar graph can be used to control the performance of the system under a particular distribution of the repair personnel while the system is in the operational phase.

\subsubsection{Buffer Optimization}

Optimizing the allocation of buffer capacity in a production line is a way to improve the system performance involving structural layout modifications. The role of buffers is to partially protect machines from the propagation of failures throughout the line, limiting the effect of blocking and starvation phenomena. The throughput curve as a function of the buffer capacity for the real system under analysis was shown in Figure 11(b). As it was noticed, an increment in the buffer capacity always leads to an increment in the system throughput. However, buffer modules represent a cost in terms of WIP and equipments. Therefore, it becomes crucial to correctly distribute the buffer capacity in the line. In the literature, two relevant problems dealing with the allocation of buffers in production lines have been formulated and solved, namely the primal and the dual problems (Gershwin and Schor 2000). The primal problem aims at minimizing the buffer capacity needed to meet a given target production rate. The dual problem aims at distributing in the line a given total buffer space with the objective of maximizing the system throughput. This approach finds practical application only in case of modularity of buffers. In the following, the method proposed in Colledani et al. (2005a) is used to address and solve the buffer allocation problem for the D12 line. For both formulations, two sub-problems are solved, obtained keeping or relaxing the constraint over the capacity of the two long buffers installed in the line for layout and maintenance reasons (Section 2). Indeed, a modification of the capacity of these two long buffers means a relevant change in the whole line layout, with no negligible implementation times and costs. However, interesting information for the line manager includes the additional throughput increment achievable through this modification.

The mathematical formulation of the dual problem follows.

$$
\text { Choose } \quad N=\left[\begin{array}{llll}
N_{1} & N_{2} & \ldots & N_{K-1}
\end{array}\right]
$$

$$
\text { To } \max T H(N)
$$

$$
\text { Subject to } \quad N^{\text {tot }}=\sum_{k=1}^{K-1} N_{k}, N_{k} \geq N^{m i n}, N_{k} \in Z^{+}
$$

The results of the dual buffer optimization problem are reported in Table 2. As it can be noticed, the optimal allocation provides an extremely relevant increment in throughput $(+7.32 \%)$. Such performance is much higher $(+16.87 \%)$ if the constraint over the long conveyor capacities is relaxed. This means that, even if the two long buffers provide benefits during the maintenance operations, they are not properly exploited during the system operating conditions and their capacity should be reduced.

Figure 19 and Figure 20 report the comparison between the current buffer allocation and the optimal one, respectively in the constrained and in the not constrained cases. As it can be noticed in Figure 19, the capacity of the two long conveyors does not change, while the capacity of all the other buffers is modified in the optimal configuration. From this figure, a reduction of $44 \%$ of the capacity of the buffer 
Table 2. Increment in throughput (TH) obtainable with an optimal allocation of the current buffer capacity and constraining or modifying the capacity of the two long buffers.

\begin{tabular}{lcc}
\hline & TH & Additional Total Buffer Capacity \\
\hline Constrained & $+7.32 \%$ & $0 \%$ \\
NOT constrained & $+16.87 \%$ & $0 \%$ \\
\hline
\end{tabular}

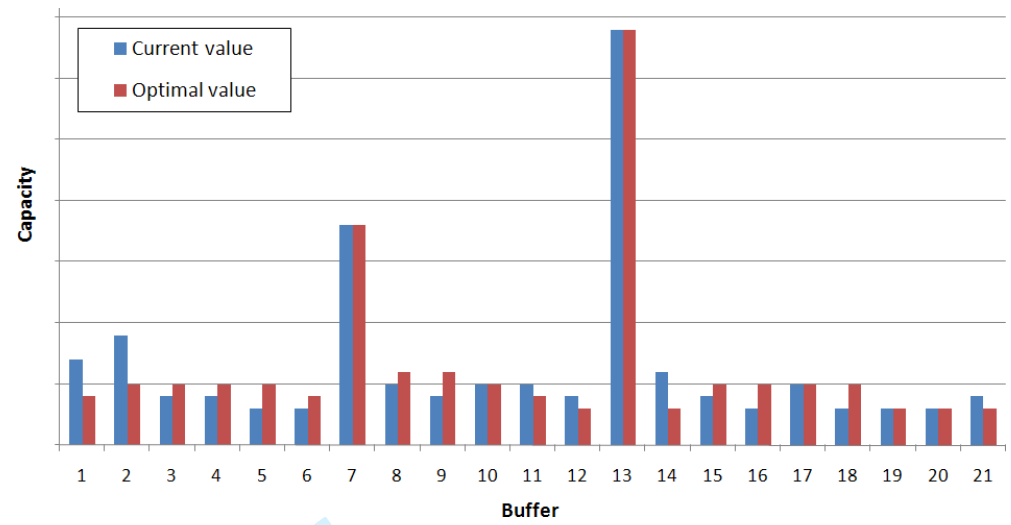

Figure 19. Optimal allocation of the current buffer capacity under a constraint over the capacity of the two long buffers. Values are not reported for confidentiality reasons.

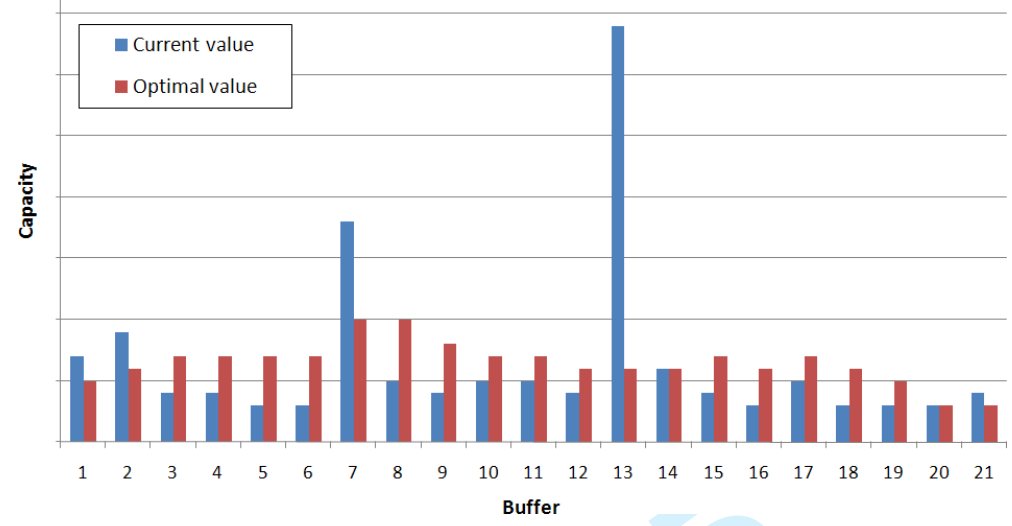

Figure 20. Optimal allocation of the current buffer capacity by relaxing the constraint over the capacity of the two long buffers. Values are not reported for confidentiality reasons.

between operation 080 and $090\left(B_{7}\right)$ and a reduction of $82 \%$ of the capacity of the buffer between operation 140 and $150\left(B_{13}\right)$ could be achieved, according to the optimal solution. Moreover, these results confirm the insights derived while studying the blocking and starvation probability propagation in Section 4.4.5. In Figure 20 it can be noticed that the capacity of the buffer downstream operation $090\left(B_{7}\right)$ should be doubled as well as the capacity of the buffer downstream operation 190 $\left(B_{18}\right)$.

The mathematical formulation of the primal problem follows.

$$
\begin{aligned}
& \text { Choose } \quad N=\left[\begin{array}{lll}
N_{1} & N_{2} \ldots N_{K-1}
\end{array}\right] \\
& \text { To } \min N^{t o t}=\sum_{k=1}^{K-1} N_{k} \\
& \text { Subject to } T H(N) \geq T H^{O b j}, N_{k} \geq N^{m i n}, N_{k} \in Z^{+}
\end{aligned}
$$

The results (Table 3 ) show that it would be possible to meet the same throughput as that of the system in the actual configuration with a $10 \%$ saving in the total 
of the not constrained optimization, where the capacity of the two long buffers can be reduced. It is also interesting to highlight that, in order to meet the target throughput of the system (20\% larger than the actual one), only an additional $7 \%$ buffer capacity is needed, in the optimal configuration of the system (Table 4).

Table 4. Increment in the total buffer capacity to get the target throughput of the line (20\% higher than the current one) both constraining and relaxing the capacity of the two long buffers.

\begin{tabular}{lcc}
\hline & TH & Additional Total Buffer Capacity \\
\hline Constrained & $+20.02 \%$ & $+24 \%$ \\
NOT constrained & $+20.03 \%$ & $+7 \%$ \\
\hline
\end{tabular}

The figures provided by the solution of the buffer allocation problem, under all its formulations, are used by the company to evaluate the convenience of a system structural re-configuration, also involving the reconfiguration and equipment cost in the analysis.

\subsection{Improvement Action Implementation}

The implementation of the identified improvement actions closes the improvement loop. Among the different options, the company can select the most favorable ones according to the involved capital and its internal improvement strategy. In particular, those actions not implying relevant costs, such as the repair crew reallocation and the priorities of intervention during simultaneous failures, were taken according to the results presented in this project. Regarding the buffer allocation, a slight modification of the system was introduced involving the increment in the storage space of the most critical buffers. However, due to the costs involved in the reconfiguration, no buffer capacity was reduced.

\section{Impact on Scania}

This new approach for manufacturing system continuous productivity improvement was perceived by Scania's manufacturing research team as an high value added methodology to be used both at a shop floor level, as a control tool, and at a management level, as an improvement tool. In particular, its strength was identified in supporting the decision making process with analytical tools able to select among different alternatives of improvement and to prove and justify the optimality of the adopted solutions. Furthermore, it was the first time that such an accurate analysis on the data collected by the system supervisor was carried out in the company. This also gave the company a feedback about the quality of the data stored in the database and hints on how to improve the monitoring system. 


\section{Conclusions}

The collaboration between Politecnico di Milano, Kungliga Tekniska högskolan and Scania allowed a high increment in the performance of the 6-cylinder engine-block line. It also enabled the formalization of a new approach for general manufacturing system performance improvement.

The different steps of the methodology have been presented along with the related results. The performance evaluation was performed through accurate techniques based on real data directly collected from the workshop. Improvement actions were defined analytically and tested on the virtual model before being implemented. The repair priority assignment and the repair crew optimization have been proposed as organizational improvements, while the optimal allocation of the buffer capacity involved structural modifications. The impact of these reconfigurations solutions on the system productivity performance measures have been quantitatively evaluated. The results of this analysis enable further economic considerations that are internal to Scania and may not be disclosed for confidentiality reasons.

The methodology, used in this case during the running phase of the manufacturing system, is thought to support decision making also in configuration and reconfiguration problems that are more and more frequent in nowadays variable environment. The D12 line has in fact been recently modified with the addition of three new operations in order to machine a block for a new low emission engine, in addition to the process described here. Further developments are in process with the intention of providing the company with a computer based tool integrated with the decision support system and usable at different times during the system life-cycle to ensure its competitiveness. Furthermore, an advanced economic model will be developed in order to evaluate, in economic terms, the relevance of the proposed actions with respect to the long term horizon and taking into consideration uncertainty in the manufacturing context.

\section{Acknowledgements}

This research has been partially funded by $V R L-K C i P$ (www.vrl-kcip.org), a Network of Excellence in the $6^{\text {th }}$ Framework Program of the European Commission. A special acknowledgment goes to Scania.

The authors would like to thank the anonymous reviewers who contributed to improve the quality of this paper.

\section{References}

Alden, J.M., Burns, L.D., Costy, T., Hutton, R.D., Jackson, C.A., Kim, D.S., Kohls, K.A., Owen, J.H., Turnquist, M.A. and VanderVeen, D.J., 2006. General Motors increases its production throughput. Interfaces, 36 (1), 6-25.

Almgren, H., 2000. Pilot production and manufacturing start-up: the case of Volvo S80. International Journal of Production Research, 38 (17), 4577-4588.

Bley, H. and Wuttke, C.C., 1997. Distributed simulation applied to production systems. Annals of the CIRP, 46 (1), 361-364.

Burman, M.H., Gershwin, S.B. and Suyematsu, C., 1998. Hewlett-Packard uses operations research to improve the design of a printer production line. Interfaces, 28 (1), 24-36.

Buzacott, J.A., 1967. Automatic transfer lines with buffer stocks. International Journal of Production Research, 5, 183-200. 
Chiang, S.Y., Kuo, C.T., Lim, J.T. and Meerkov, S.M., 2000. Improvability of assembly systems I: Problem formulation and performance evaluation. Mathematical Problems in Engineering, 6 (4), 321-357.

Colledani, M., Gandola, F., Matta, A. and Tolio, T., 2008. Performance evaluation of linear and non linear multi-product multi-stage lines with unreliable machines and finite homogeneous buffers. IIE Transactions, 40 (6), 612-626.

Colledani, M., Grasso, M., Matta, A. and Tolio, T., 2005a. A new analytical method for buffer space allocation in production lines. CIRP Journal of Manufacturing Systems, 34 (4).

Colledani, M., Matta, A. and Tolio, T., 2005b. Performance evaluation of production lines with finite buffer capacity producing two different products. $O R$ Spectrum, 27, 243-263.

Colledani, M., Moriggi, P., Polato, A., Lundholm, T. and Tolio, T., 2007. A decomposition method to support evaluation and continuous improvement of reconfigurable manufacturing system performance. $40^{\text {th }}$ CIRP International Seminar on Manufacturing Systems, 30 May - 1 June 2007, Liverpool, UK.

Colledani, M. and Tolio, T., 2005. A decomposition method to support the configuration/reconfiguration of production systems. CIRP Annals, 54 (1), 441-444.

Commault, C., Dallery, Y. and Frein, Y., 1996. Modeling and analysis of closed loop production lines with unreliable machines and finite buffers. IIE Transactions, $545-554$.

Dallery, Y., David, R. and Xie, X.L., 1988. An efficient algorithm for analysis of transfer lines with unreliable machines and finite buffers. IEEE Transactions, 20(3), 280-283.

DeVin, L.J., Oscarsson, J., Ng, A., Jägstam, M. and Karlsson, T., 2004. Manufacturing simulation: good practice, pitfalls, and advanced applications. $21^{\text {st }}$ International Manufacturing Conference, 1-3 September 2004, Limerick, Ireland, 156-163.

Diamantidis, A.C., Papadoupulos, C.T. and Vidalis, M.I., 2004. Exact analysis of a discrete material three-station one-buffer merge system with unreliable machines. International Journal of Production Research, 42, 651-675.

Ferdinand, A.E., 1971. An analysis of the machine interference model. IBM Systems Journal, 10 (2), 129-142.

Freiheit, T., Wang, W. and Patrick, S., 2007. A case study in productivity-cost trade-off in the design of paced parallel production systems. International Journal of Production Research, 45 (14), 3263-3288.

Gershwin, S.B., 1987. Representation and analysis of transfer lines with machines that have different processing rates. Annals of Operations Research, 9, 511530.

Gershwin, S.B., 1994. Manufacturing Systems Engineering. Prentice Hall.

Gershwin, S.B. and Burman, M.H., 2000. A Decomposition method for analysing inhomogeneous assembly/disassembly systems. Annals of Operations Research, 93, 91-116.

Gershwin, S.B., Matta, A. and Tolio, T., 2002. Analysis of two-machine lines with multiple failure modes. IIE Transactions, 34, 51-62.

Gershwin, S.B. and Schor, J.E., 2000. Efficient algorithms for buffer space allocation. Annals of Operations Research, 93, 117-144.

Gershwin, S.B. and Werner, L.M., 2003. An approximate analytical method for evaluating the performance of closed loop flow systems with unreliable machines and finite buffers (large loops). MIT Laboratory for Manufacturing and Productivity Report.

Gopalan, M.N. and Kumar, U.D., 1995. On the utilization of a split production 
system with inter-stage inspection. International Journal of Production Economics, 38 (2-3), 107-116.

Haque, L. and Armstrong, M.J., 2007. A survey of the machine interference problem. European Journal of Operational Research, 179 (2), 469-482.

Helber, S., 1999. Performance analysis of flow lines with non-linear flow of material. Springer.

Koster, R.D. and Wijngaard, J., 1989. Continuous vs. discrete models for production lines with blocking. In: H.G. Perros and T. Altiok, eds. Queuing networks with blocking, Elsevier Science Publishers, 175-191.

Kuhn, H., 2003. Analysis of automated flow line systems with repair crew interference. In: S.B. Gershwin, Y. Dallery, C.T. Papadopoulos and J.M. Smith, eds. Analysis and Modeling of Manufacturing Systems, Kluwer Academic Publishers, $155-179$.

Li, J., 2003. Modelling and analysis of complex production systems. $4^{\text {th }}$ Aegean International Conference in Analysis of Manufacturing Systems, 1-4 July 2003, Samos Island, Greece, 200-210.

Li, J. and Huang, N., 2005. Modelling and analysis of a multiple product manufacturing system with split and merge. International Journal of Production Research, 43, 4049-4066.

Liberopoulos, G. and Tsarouhas, P., 2002. Systems analysis speeds up Chipita's food-processing line. Interfaces, 32 (3), 62-76.

Matta, A. and Tolio, T., 1998. A method for performance evaluation of automated flow lines. CIRP Annals, 47 (1), 373-376.

Nyhuis, P., von Cieminski, G., Fischer, A. and Feldmann, K., 2005. Applying simulation and analytical models for logistic performance prediction. CIRP Annals, 54 (1), 417-422.

Nyhuis, P. and Wiendahl, H.P., 2006. Logistic production operating curves - Basic model of the theory of logistic operating curves. CIRP Annals, 55 (1), 441-444.

Patchong, A., Lemoine, T. and Kern, G., 2003. Improving car body production at PSA Peugeot Citroën. Interfaces, 33 (1), 36-49.

Phillis, Y.A., Sourlas, D., Kouikoglu, V.S. and Manousiouthakis, V., 1997. Design of serial production systems using discrete event simulation and nonconvex programming techniques. International Journal of Production Research, 35, $753-766$.

Savsar, M. and Al-Jawini, A., 1995. Simulation analysis of just-in-time production systems. International Journal of Production Economics, 42, 67-78.

Shiue, G.A. and Altiok, T., 1993. Two-stage, multi-product production/inventory systems. Performance Evaluation, 17, 225-232.

Stecke, K. and Aron, J., 1985. Review of operator/machine interference models. International Journal of Production Research, 23, 129-151. 